Spatially resolved analysis of ion migration dynamics in metal halide perovskite single crystals

Juan F. Galisteo-López, * Mauricio E. Calvo, Hernán Míguez*

Instituto de Ciencia de Materiales de Sevilla, Consejo Superior de Investigaciones Científicas (CSIC)-Universidad de Sevilla, C/Américo Vespucio 49, 41092 Sevilla, Spain 
ABSTRACT:

The spectacular advances in efficiency of optoelectronic devices based on hybrid organic-inorganic lead-halide perovskites have been accompanied by detailed structural and optical studies in order to shed some light on the instability presented by these materials, which constitute their main bottleneck for commercialization. Following a pump and probe scheme in a laser scanning confocal microscope we are able to resolve the characteristic photo-induced emission activation/deactivation dynamics in methyl ammonium lead bromide single crystals with millisecond and sub-micrometre temporal and spatial resolution. This information is complemented with a detailed study of spectral variations and the observed changes are interpreted in the framework of lightinduced ion migration and associated defect passivation taking place mainly within the bulk of the material. Our results point to the presence of photo-induced structural changes likely accompanying the migration of ions.

\section{ToC Graphic:}
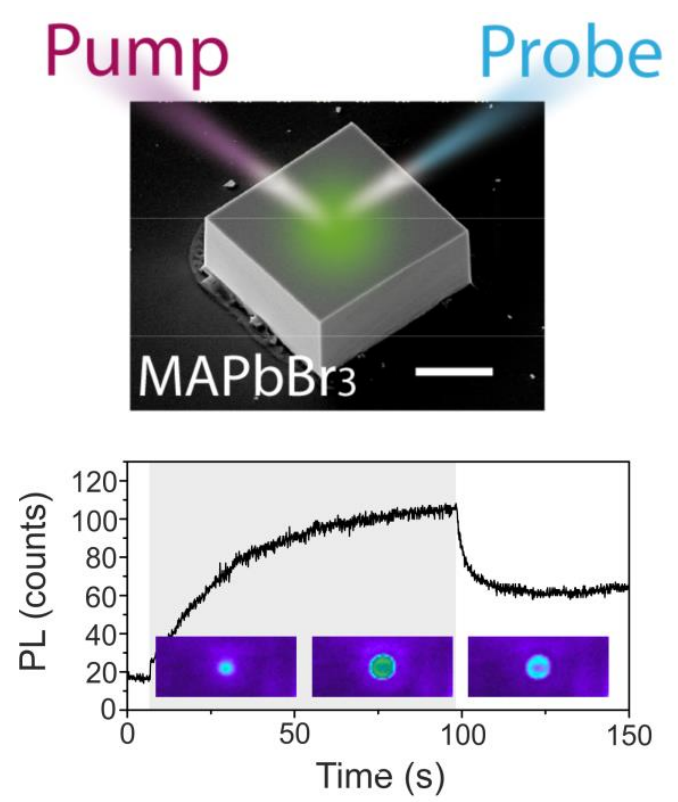
Over less than a decade hybrid organic inorganic perovskites have brought third generation photovoltaics to efficiencies competing with those of silicon solar cells. [1] Further, these materials have evidenced potential in several other fields such as a light emission, [2,3] displays, [4] X-ray imaging [5] or sensing. [6,7] While spectacular progress has been made in a short time span, some issues remain regarding these materials which constitute a bottleneck when applications are considered. The presence of lead in the best performing materials for photovoltaic devices poses a serious environmental concern [8] and lead-free alternatives are still well behind in terms of efficiency. [9] On the other hand, the lack of stability of the perovskite matrix against environmental and thermal conditions $[10,11,12,13]$ as well as irradiation needs to be tackled in order to deliver stable long-lasting materials demanded by the industry. In this respect, intense research efforts have brought photovoltaic devices to one-year stable operation [14] which represents a giant leap with respect to initially reported values but need to be improved to the 25 year lifetime of commercial silicon solar cells.

Instability under irradiation represents a crucial issue bearing in mind that most uses envisaged for this material deal with its interaction with electromagnetic radiation. When illuminated over prolonged periods of time $[15,16,17,18]$ or with relatively high intensities $[19,20,21]$ these materials undergo an irreversible physical degradation which renders them useless for any of the above mentioned applications. On the contrary, when illuminated with low intensities over small periods of time, hybrid organic inorganic perovskites improve their emission on a seconds-minutes time lapse. $[22,23,24,25,26,27,28,29,30,31,32]$, One key ingredient in this photo-activation is the role of the atmosphere, where the presence of oxygen favors such photoluminescence (PL) enhancement. [24,25,26,28,31,33] Increasing evidence of the presence of ion migration in the photo-activation [27] and/or degradation [15,20,21] of these materials 
has appeared, pointing to a connection with the photo-instability characteristic of mixed-halide perovskites. [34] In this direction several approaches have been presented to improve the stability such as the use of mixed cation and mixed anion compositions $[35,36]$ where an increased formation energy for structural defects such as vacancies [37] can lead to a more stable PL as ion migration needs from this type of defect to take place. Approaches to further improve the photostability in these samples have focused on eliminating surface halide-related traps, and thus reducing ionic movement, either modifying the synthesis [38] or employing post-synthetic treatments involving a combination of light irradiation and humid air [39].

Recent reports have proposed different mechanisms as the driving force behind the ion migration causing photo-activation, such as the annihilation of photo-excited Frenkel pairs [32] or, taking into account the role of the atmosphere, the interaction of atmospheric oxygen with halide-related defects such as vacancies [39] and interstitials [40] or the formation of a layer of negatively charged species that adsorb on the semiconductor surface as a result of photoexcitation in the presence of oxygen. [41] In this last mechanism, the presence of the surface charge accounts for both photoactivation as well as material degradation, evidencing the concurrence of both processes under exposure to light and oxygen. Once the underlying mechanism for this photoinstability has been revealed, an analysis of the dynamics of ion migration occurring under illumination is in order, as its control could help preventing degradation while allowing photoactivation.

To date most studies on the photo-activation or photo-degradation dynamics are usually performed on polycrystalline films as they are more easily fabricated and represent the morphology of choice in photovoltaic devices. But this usually complicates the interpretation of results as intrinsic processes taking place within a crystal are combined 
with those related with grain boundaries and other types of defects which abound in polycrystalline films. Ideally one would like to isolate those effects which arise from the interaction of light and moving ions with a well-defined crystalline lattice, amenable to be theoretically modelled, in order to understand them before generalizing them to the more realistic, albeit hard to model, case of the polycrystalline film.

In this work we present a photophysical study of large (thousands of cubic microns) methyl ammonium lead bromide $\left(\mathrm{CH}_{3} \mathrm{NH}_{3} \mathrm{PbBr}_{3}\right)$ single crystals. With the simultaneous use of two optical excitation sources in a laser scanning confocal microscope (LSCM) and a high speed camera we study the dynamics of PL activation/deactivation in these structures following a pump and probe procedure which allows a high temporal (millisecond) and spatial (sub-micron) resolution. After performing several activation/deactivation cycles we observe how, upon illumination, the dynamics of the emission, as well as its spectral response, are modified. We discuss the possible origin of the observed changes in terms of the migration of halide ions in the presence of vacancy/interstitial defects and how they are affected by light-induced structural changes.

$\mathrm{CH}_{3} \mathrm{NH}_{3} \mathrm{PbBr}_{3}$ single crystals, having cuboid shapes and dimensions of thousands of $\mu \mathrm{m} 3$ (see Fig.1a), were synthesized following an already reported method. [42] The photophysics of the crystals were studied with a LSCM microscope (Zeiss LSM 700 Duo). A dual illumination scheme (using a Zeiss Plan-Apochromat 20x NA=0.8 M27 objective) was employed as follows: while a selective high intensity irradiation ( $\mathrm{P}=34$ $\mathrm{W} / \mathrm{cm} 2, \lambda=405 \mathrm{~nm})$ was performed on a reduced spatial region $(1.66 \mu \mathrm{m}$ diameter $)$, the PL from a larger region $(40 \times 40 \mu \mathrm{m})$ was imaged with a lower intensity $(\mathrm{P}=0.28 \mathrm{~W} / \mathrm{cm} 2$, $\lambda=488 \mathrm{~nm}$ ) beam. The illumination was carried out in a pump-probe scheme in which only the PL signal originating from the less intense probe was collected. In order to do 
so, during the photo-activation, the high intensity pump is performed over ca. 1 minute with $17 \mathrm{~ms}$ intervals and a duty cycle of $18.75 \%$. The PL from the low intensity probe is collected only when the high intensity pump is not active. The intensity of the probe beam was chosen so that no change in PL was observed on the sample when measured over 5 minutes. In this way the local changes induced by the high intensity pump were monitored in real time. The sample was illuminated through the side in contact with the glass substrate. The LSCM is equipped with a spectral CCD (spectral resolution of 4.9 $\mathrm{nm}$ ) so that the spectral properties of the sample could be probed before and after the selective high intensity irradiation.

First we study the PL evolution of the single crystals, considering a large sample region comprising the optically pumped one together with a reference one (see Fig.1b,c,d). Here it is clearly seen how the PL of the sample monotonically grows as it is optically excited and then stabilizes. In order to ensure that the probe beam is not introducing any effect on the PL dynamics, PL from a similar region located $10 \mu \mathrm{m}$ away from the pumped one (see Fig.1b) was collected and no changes were observed. As the pump beam is removed the PL drops exponentially to a level above that of its initial PL. If the sample is optically pumped again it undergoes a new activation over a time scale below $17 \mathrm{~ms}$, given by the resolution of the instrument. Further de-activation/activation steps take place in this manner following an exponential decay and an instant activation respectively. When probing the $\mathrm{PL}$ evolution of smaller (500 $\mathrm{nm}$ diameter) regions located close to the center (Fig.1e,f) and edge (Fig.1g,h) of the illuminated region we see a similar behavior only that activation dynamics vary, while deactivation ones seem to remain constant. A detailed analysis will be presented below. 

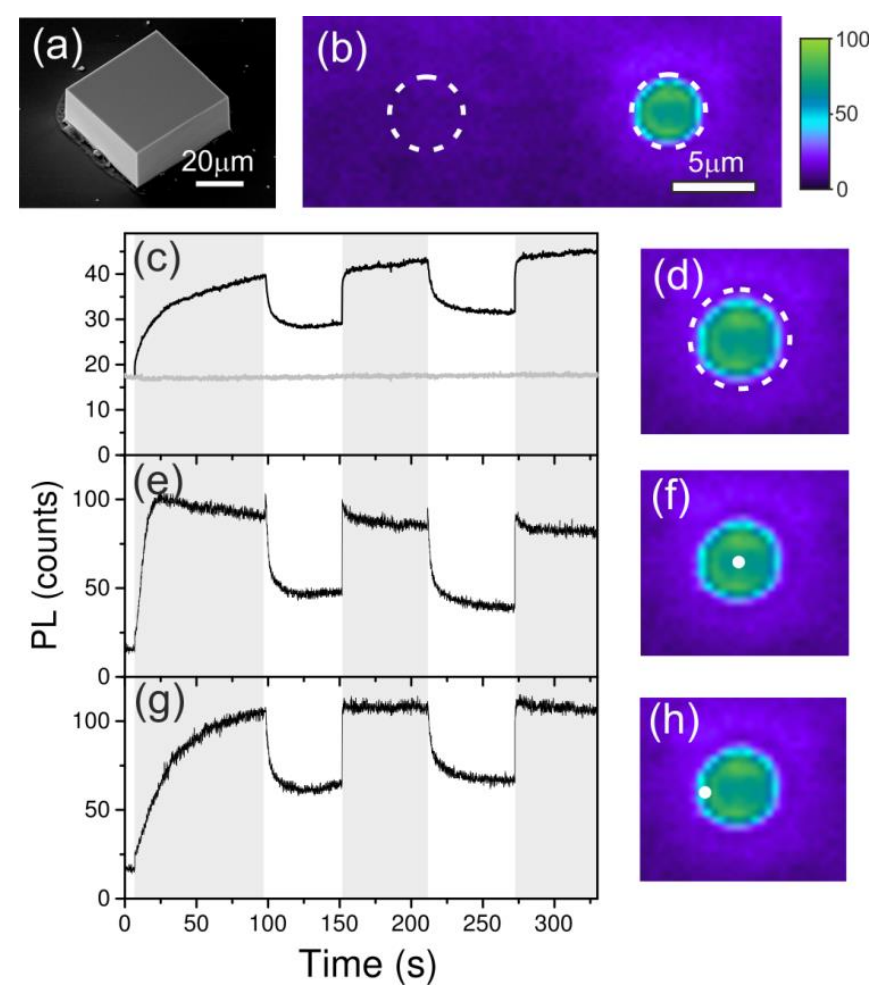

Figure 1. (a) $\mathrm{SEM}$ image of a $\mathrm{CH}_{3} \mathrm{NH}_{3} \mathrm{PbBr}_{3}$ crystal like the ones under study. (b) PL image showing the optically pumped region and a reference one. (c) PL dynamics of a single crystal collected from a large region, highlighted as a dashed circle in (d) as an intense pump is focused on its surface (grey panels) and removed (white panels). Dark grey curve represents a reference PL collected from a region $10 \mu \mathrm{m}$ away from the illuminated one. Similar data collected from a smaller $(0.5 \mathrm{x} 05 \mu \mathrm{m})$ region close to the center and edge of the pumped region are shown in $(e, f)$ and $(g, h)$ respectively.

Next we consider the spatial variation of the PL in the surroundings of the optically excited region. Fig.2a-c represent PL images collected at three different time steps corresponding to the initial rise of the activation (12s), its stabilization (75s) and the deactivation (150s). Together with the PL images, emission profiles taken from them are shown in Fig.2d-f. For the latter, the background PL was subtracted in order to show only the enhancement during optical pumping. In the initial stages of the activation (Fig.2a,d) we observe a spatial region with enhanced PL which coincides with that of 
the illuminated region. As the excitation continues, (Fig.2b,e) the center of the pumped region reaches a maximum PL and slightly decreases while the adjacent corona is further enhanced. This is better appreciated if one considers the time evolution of the PL at the center and edge of the illuminated region (see Fig.1e-f and g-h). Finally, when the pump beam is removed, the whole region presents a drop in PL but remains activated above the initial PL levels (Fig.2c,f). This residual activation is clearly stronger in the corona surrounding the center of the illuminated region. An animation, constructed using the PL images taken by the CCD camera every $17 \mathrm{~ms}$, is included as Supporting Information and shows the temporal and spatial evolution of the photoemission of a region of circa $20 \mu \mathrm{m}$ when only a circular region of $1.6 \mu \mathrm{m}$ diameter in its center is pumped.
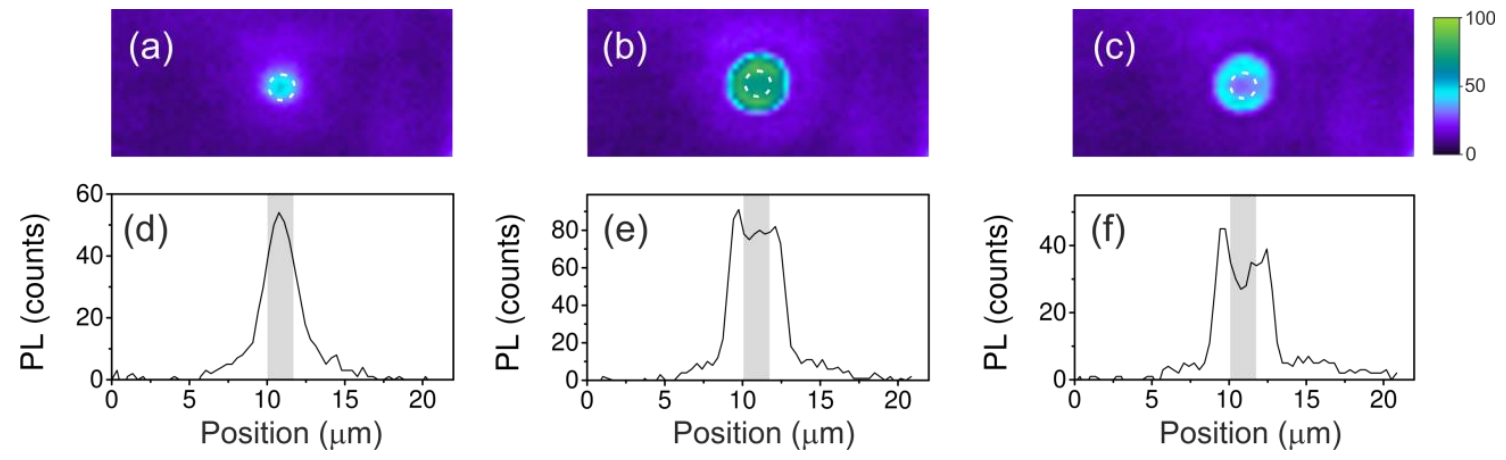

Figure 2. (a-c) PL images of the illuminated area at three different steps of the dynamics: 12s (a), 75s (b) and 150s (c). The dashed circle shows the pumped region. (df) Emission profiles extracted from the PL images across a horizontal line crossing the center of the illuminated region. Grey panels show the pumped region. Scale of PL images (a-c) is identical to that of (d-f).

In order to gain information on the activation/de-activation process of the emission we extracted PL dynamics from a series of $0.5 \times 0.5 \mu \mathrm{m}$ regions (like the ones shown in Fig.1e-f and g-h) along a line crossing the illuminated region (Fig.3). Fig.3a shows 
typical emission profiles during the PL activation and when it is completed. The initial activation was best fitted to an exponential growth:

$$
I_{P L}=I_{P L}^{0}+A \cdot\left(1-e^{-t / t_{a}}\right)
$$

Where $t_{a}$ is the activation time from which we extracted the activation rate $k_{a}=1 / t_{a}$ as a function of position on the sample (see Fig.3b). Here it is evident how the activation follows well the emission profile, where the spatial regions presenting the larger PL enhancement coincide with those being more rapidly activated with maxima $k_{a}=0.23 \mathrm{~s}^{-}$ 1. Next we fitted the first de-activation part of the PL dynamics for the above points (as no difference was found between this and successive de-activations) to an exponential decay and extracted the de-activation rate $k_{d}$ (Fig. 2c). Here it can be clearly seen how $k_{d}$ presents smaller variations across the illuminated region.
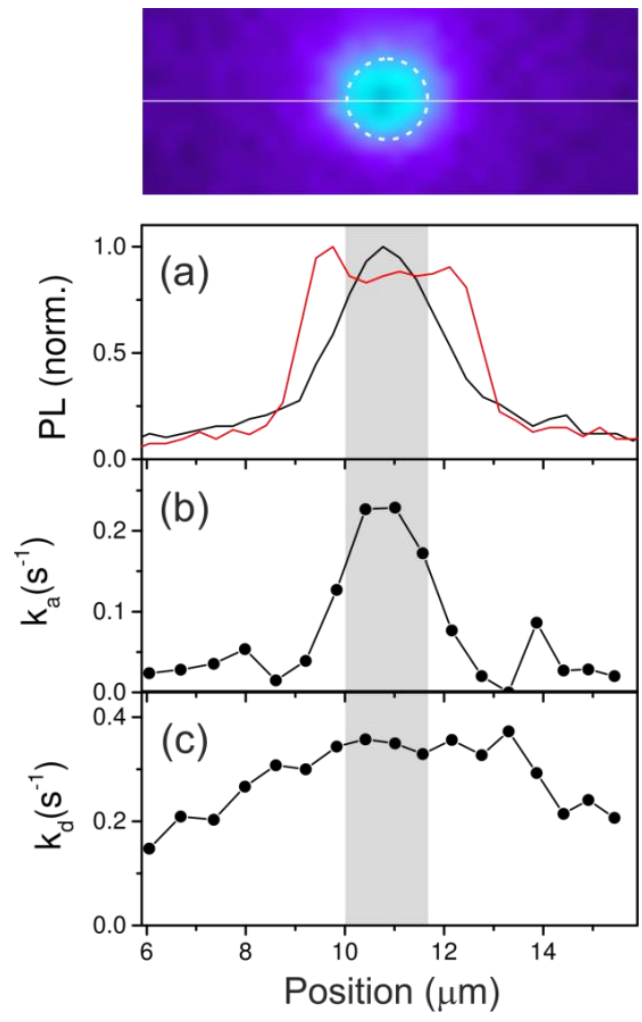

Figure 3. (a) Normalized PL profiles of the sample during the activation process (black curve) and when it has arrived at a stationary behavior (red curve). (b) and (c) show the 
spatial evolution of the activation $\left(\mathrm{k}_{\mathrm{a}}\right)$ and deactivation $\left(\mathrm{k}_{\mathrm{d}}\right)$ constants across a line going through the illuminated region (solid line in the top image). Grey panel shows the spatial region where the sample is optically pumped.

Finally the spectral properties of the illuminated region and its surroundings were studied by employing the spectral CCD camera. In order to do so, a spectral image was taken before and after the sample was photo-activated with the high intensity pump. Figure 4a shows PL spectra from a region of the sample before and after performing a high intensity optical pump as mentioned above. Here it is evident that upon optically pumping the sample, and hence photo-activating it, the PL from that region is blueshifted. To appreciate the spatial distribution of the spectral changes, Fig. $4 \mathrm{~b}$ shows a spectral analysis over a large region comprising the illuminated one. The above mentioned blue-shift is evident in the illuminated region while the rest of the structure remains spectrally unchanged. If we now compare a profile of the spectral changes across the illuminated region (Fig.4c) with a PL profile one we observe a nearly identical trend, further evidencing the relationship between the activation and the spectral changes. 

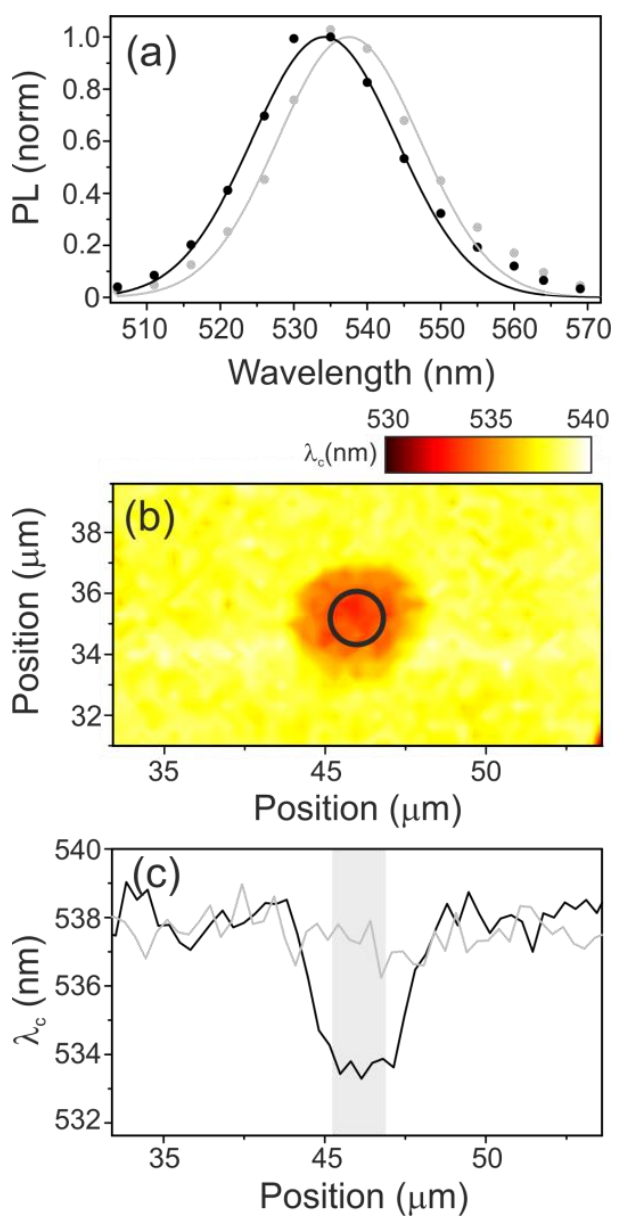

Figure 4. (a) Normalized PL spectra of a sample region before (grey) and after (black) photo-activation. Points (lines) correspond to experimental data (and their fit to a Gaussian curve). (b) Spectral PL image of a sample region comprising the illuminated one (marked by the black circle). (c) Spectral profiles across a line in the region shown in (b) before (grey) and after (black curve) photo-activation. Grey box indicates spatial region where the optical pumping is performed.

Changes in the spatial distribution of halide ions under external visible light illumination have been reported for different metal-halide perovskites. [21,27] Further, the slow dynamics shown above for the PL agree with photocurrent activation and deactivation transients previously observed in metal-halide perovskite solar cells and associated with the migration of halide ions under an external applied field. $[43,44,45]$ 
Also similar dynamics and changes in the spatial distribution of halide species monitored through PL have been observed in $\mathrm{CH}_{3} \mathrm{NH}_{3} \mathrm{PbI}_{3}$ films under external applied electric fields. $[46,47,48]$ Thus we assume that the observed changes in PL are caused by a spatial redistribution of halide ions. The initial activation dynamics taking place during the first tens of seconds evidence how under the presence of external irradiation halide ions acquire the necessary energy to migrate. As the enhancement in emission in the illuminated region is not accompanied by any darkening of the surroundings it seems that under this level of external illumination migration of vacancies, acting as deep traps quenching $\mathrm{PL}$ in $\mathrm{CH}_{3} \mathrm{NH}_{3} \mathrm{PbBr}_{3}$, [49] to nearby interstitial ions is induced causing the annihilation of defects which leads to a PL enhancement. [50] This picture matches the one recently proposed by Mosconi and co-workers for $\mathrm{CH}_{3} \mathrm{NH}_{3} \mathrm{PbI}_{3}$ [32] involving the annihilation of optically excited Frenkel pairs formed by iodide vacancies/interstitials present in the material. The driving force for the migration of negatively charged interstitial halide defects is also provided by the formation of negatively charged superoxide layers at the surface of the material as a consequence of the light-induced formation of superoxide species as mentioned above. [41] Both mechanisms are expected to be further enhanced due to an increased ionic conductivity in these materials under illumination. [51] According to the measured dynamics, the activation rates (see Fig.3b) depend on the pump intensity as the spatial variation of $k_{a}$ follows that of the illuminated region. This fact was further proved by studying the evolution of the maximum value of $k_{a}$, taking place at the center of the illuminated region, as a function of the pump intensity and obtaining a linear relationship.

Once the external high intensity pump is switched off, the sample PL drops to a point above its initial emission (see Fig.1). This fact points to structural changes taking place within the $\mathrm{CH}_{3} \mathrm{NH}_{3} \mathrm{PbBr}_{3}$ lattice during irradiation. While upon removal of the high 
intensity pump many defects seem to form again, as evidenced by the exponential decay of PL taking place in tens of seconds, the fact that the residual PL is above the initial one points to a population of annihilated defects remaining in a metastable state. As suggested in Ref. 32 this could be due to entropic differences between the high temperature synthesis conditions and the room temperature PL experiments. In the picture of Frenkel-pair annihilation under optical excitation the observed behavior, with a partial recovery of the PL within tens of seconds and a fully one within hours, indicates the existence of two populations of defects. The difference between the two populations could be related with the separation between the halide vacancy and the interstitial which is known to affect the formation and annihilation energies of these defects in semiconductors. [52]

The de-activation rate back to the meta-stable state $k_{d}$ is now less dependent on spatial position, (Fig.3c) as expected, since Frenkel pairs annihilated during light illumination should not keep memory on whether the process was carried out through a high or low intensity irradiation. In this new metastable state, subsequent light-induced annihilation of the defect takes place at a much lower energetic cost. This is evidenced by the fact that upon further optical excitation the PL is re-activated over times below the resolution of our measurement (that is, up to 3 orders of magnitude faster than the initial activation). This point would indicate that upon the initial photo-induced activation the metal-halide crystal has undergone structural changes which lower the activation energy for the migration of the halide ions. Further evidence for such structural changes comes from the spectral blue-shift taking place in the sample region which has been photoactivated. Previous reports of blue-shifted $\mathrm{PL}$ near the edges of $\mathrm{CH}_{3} \mathrm{NH}_{3} \mathrm{PbI}_{3}$ single crystals [53] have been associated with a deformation of the crystalline lattice. While in the latter case the lattice deformation was due to the interaction of the halide crystal 
edge with atmospheric water, in our case the lattice distortion is induced by the external high intensity illumination and the migration of halide ions it drives. As the activation energy for ion migration depends, among other factors, on the crystal structure, a lattice deformation could influence the activation rate under illumination and also introduce migration channels. [54] In this direction calculations by Gottesman and co-workers, [55] carried out to account for photocurrent transients with similar duration to the activation/deactivation presented here, showed that in the presence of illumination and bias the lattice structure of $\mathrm{CH}_{3} \mathrm{NH}_{3} \mathrm{PbI}_{3}$ could undergo a deformation. Finally it should be mentioned that the nature of these changes is different from recent reports of lightinduced lattice expansion as recently reported for $\mathrm{CH}_{3} \mathrm{NH}_{3} \mathrm{PbI}_{3}$ single crystals and related mixed-cation perovskites [56, 57] which would lead to a redshift of the emission.

In summary, the time and spatially resolved photo-activation and deactivation dynamics of $\mathrm{CH}_{3} \mathrm{NH}_{3} \mathrm{PbBr}_{3}$ single crystals have been studied. The changes in emission, assumed here to be due to the migration of halide ions under external illumination, point to a complex scenario where two populations of defects are present with different activation energies which are annihilated during illumination and restored in the dark with two different time scales. Further, upon illumination, structural changes in the perovskite lattice, evidenced by spectral changes in the material emission, take place and affect the migration of ions upon successive activation processes. While, in principle, the instability could be reduced by improving the fabrication procedure in order to minimize the defect concentration, the issue of lattice deformation under illumination should be further studied as it represents a crucial bottleneck for a material expected to play a key role in future optoelectronic devices. 


\section{ASSOCIATED CONTENT}

XRD characterization of the sample employed and the fitting of the activation and deactivation dynamics. (PDF)

Video showing the effect of ion migration on activation and deactivation. This material is available free of charge via the Internet at http://pubs.acs.org.

\section{AUTHOR INFORMATION}

\section{Notes}

The authors declare no competing financial interests.

\section{Corresponding authors}

Juan F. Galisteo-López, juan.galisteo@ @sic.es

Hernán Míguez, h.miguez@csic.es

\section{ACKNOWLEDGMENT}

Financial support of the Spanish Ministry of Economy and Competitiveness under grant MAT2017-88584-R is gratefully acknowledged. Juan Luis Ribas at the Centro de Investigación Tecnología e Innovación de la Universidad de Sevilla (CITIUS) is acknowledged for assistance with confocal microscopy experiments.

\section{REFERENCES:}

1 See http://www.nrel.gov/ncpv/images/efficiency_chart.jpg for an updated list of certified efficiencies. 
${ }^{2}$ Sutherland, B. R. and Sargent, E. H. Perovskite photonic sources. Nat. Photon. 2016, $10,295-302$.

${ }^{3}$ Kim, Y-H.; Cho, H.; Lee, T-W. Metal halide perovskite light emitters. Proc. Natl. Acad. Sci. U. S. A. 2016, 113, 11694-11702.

${ }^{4}$ Gu, L.; Tavakoli, M. M.; Zhang, D.; Zhang, Q.; Waleed, A.; Xiao, Y.; Tsui, k-H.; Lin, Y.; Liao, L.; Wang, J.; Fan, Z. Adv. Mater. 2016, 28, 9713-9721.

${ }^{5}$ Wei, W.; Zhang, Y.; Xu, Q.; Wei, H.; Fang, Y.; Wang, Q.; Deng, Y.; Li, T.; Gruverman,. A.; Cao, L.; Huang, J. Monolithic integration of hybrid perovskite single crystals with heterogenous substrate for highly sensitive X-ray imaging. Nat. Photon. 2017, 11, 315-322.

${ }^{6}$ Zhuang, Y.; Yuan, W.; Qian, L.; Chen, S.; Shi, G. High-performance gas sensors based on a thiocyanate ion-doped organometal halide perovskite. Phys. Chem. Chem. Phys. 2017, 19, 12876-12881.

${ }^{7}$ Stoeckel, M-A.; Gobbi, M.; Bonacchi, S.; Liscio, F.; Ferlauto, L.; Orgiu, E.; Samorì, P. Reversible, Fast, and Wide-Range Oxygen Sensor Based on Nanostructured Organometal Halide Perovskite. Adv. Mater. 2017, 29, 1702469.

${ }^{8}$ Babayigit, A.; Ethirajan, A.; Muller, M.; Conings, B. Toxicity of Organometal Halide Perovskite Solar Cells. Nat. Mater. 2016, 15, 247-251.

${ }^{9}$ Shi, Z.; Guo, J.; Chen, Y.; Li, Q.; Pan, Y.; Zhang, H.; Xia, Y.; Huang, W. Lead-Free Organic-Inorganic Hybrid Perovskites for Photovoltaic Applications: Recent Advances and Perspectives. Adv. Mater. 2017, 29, 1605005.

${ }^{10}$ Yang, J.; Siempelkamp, B.D.; Liu, D.; Kelly, T.L. Investigation of $\mathrm{CH}_{3} \mathrm{NH}_{3} \mathrm{PbI}_{3}$ Degradation Rates and Mechanisms in Controlled Humidity Environments Using in Situ Techniques. ACS Nano 2015, 9, 1955-1963. 
${ }^{11}$ Christians, J.A.; Miranda-Herrera, P.A.; Kamat, P.V. Transformation of the Excited State and Photovoltaic Efficiency of $\mathrm{CH}_{3} \mathrm{NH}_{3} \mathrm{PbI}_{3}$ Perovskite upon Controlled Exposure to Humidified Air. J. Am. Chem. Soc. 2015, 137, 1530-1538.

12 Huang, W.; Manser, J.S.; Kamat, P.V.; Ptasinska, S. Evolution of Chemical Composition, Morphology, and Photovoltaic Efficiency of $\mathrm{CH}_{3} \mathrm{NH}_{3} \mathrm{PbI}_{3}$ Perovskite under Ambient Conditions. Chem. Mater. 2016, 28, 303-311.

${ }^{13}$ Misra, R.K.; Aharon, S.; Li, B.; Mogilyansky, D.; Visoly-Fisher, I.; Etgar, L.; Katz, E.A. Temperature- and Component-Dependent Degradation of Perovskite Photovoltaic Materials under Concentrated Sunlight. J. Phys. Chem. Lett., 2015, 6, $326-330$.

14 Grancini, G.; Roldán-Carmona, C.; Zimmermann, I.; Mosconi, E.; Lee, X.; Martineau, D.; Narbey, S.; Oswald, F.; De Angelis, F.; Graetzel, M.; Nazeeruddin, M. K. One-Year Stable Perovskite Solar Cells by 2D/3D Interface Engineering. Nat. Commun. 2017, 8, 15684.

15 Henz, O.; Zhao, Z.; Gradečak, S. Impacts of Ion Segregation on Local Optical Properties in Mixed Halide Perovskite Films. Nano Lett. 2016, 16, 1485-1490.

${ }^{16}$ Li, Y.; Xu, X.; Wang, C.; Ecker, B.; Yang, J.; Huang, J.; Gao, Y. Light-Induced Degradation of $\mathrm{CH}_{3} \mathrm{NH}_{3} \mathrm{PbI}_{3}$ Hybrid Perovskite Thin Film. J. Phys. Chem.C 2017, $121,3904-3910$.

${ }^{17}$ Akbulatov, A. F.; Luchkin, S. Y.; Frolova, L. A.; Dremova, N. N.; Gerasimov, K. R.; Zhidkov, I. S.; Anokhin, D. V.; Kurmaev, E. Z.; Stevenson, K. J.; Troshin, P. A. Probing the Intrinsic Thermal and Photochemical Stability of Hybrid and Inorganic Lead Halide Perovskites. J. Phys. Chem. Lett. 2017, 8, 1211-1218. 
${ }^{18}$ Aristidou, N.; Sanchez-Molina, I.; Chotchuangchutchaval, T.; Brown, M.; Martinez, L.; Rath, T.; Haque, S. A. The Role of Oxygen in the Degradation of methylammonium Lead Trihalide Perovskite Photoactive Layers. Angew. Chem. 2015, $54,8208-8212$.

${ }^{19}$ Merdasa, A.; Bag, M.; Tian, Y.; Källman, E.; Dobrovolsky, A.; Scheblykin, I. G. J. Super-Resolution Luminescence Microspectroscopy Reveals the Mechanism of Photoinduced Degradation in $\mathrm{CH}_{3} \mathrm{NH}_{3} \mathrm{PbI}_{3}$ Perovskite Nanocrystals. Phys. Chem. $\mathrm{C}$ 2016, 120, 10711-10719.

${ }^{20}$ Yuan, H.; Debroye, E.; Janssen, K.; Naiki, H.; Steuwe, C.; Lu, G.; Moris, M.; Orgiu, E.; Uji-I, H.; De Schryver, F. et al. Degradation of Methylammonium Lead Iodide Perovskite Structures through Light and Electron Beam Driven Ion Migration. J. Phys. Chem. Lett. 2016, 7, 561-566.

${ }^{21}$ Galisteo-López, J. F.; Li, Y.; Míguez, H. Three Dimensional Optical Tomography and Correlated Elemental Analysis of Hybrid Perovskite Micro-Structures: an Insight Into Defect-Related Lattice Distortion and Photo-Induced Ion Migration. J. Phys. Chem. Lett. 2017, 7, 5227-5234.

${ }^{22}$ Sánchez, R. S.; González-Pedro, V.; Lee, J-W.; Park, N-G.; Kang, Y. S.; Mora-Sero, I.; Bisquert, J. Slow Dynamic Processes in Lead Halide Perovskite Solar Cells. Characteristic Times and Hysteresis. J. Phys. Chem. Lett. 2014, 5, 2357-2363.

${ }^{23}$ Stranks, S. D.; Burlakov, V. M.; Leitjens, T.; Ball, J. M.; Goriely, A.; Snaith, H. J. Recombination Kinetics in Organic-Inorganic Perovskites: Excitons, Free Charge, and Subgap States. Phys. Rev. Appl. 2014, 2, 034007. 
${ }^{24}$ Galisteo-López, J.F.; Anaya, M.; Calvo, M.E.; Míguez, H. J. Environmental Effects on the Photophysics of Organic-Inorganic Halide Perovskites. J. Phys. Chem. Lett. 2015, 6, 2200-2205.

${ }^{25}$ Tian, Y.; Peter, M.; Unger, E.; Abdellah, M.; Zheng, K.; Pullerits, T.; Yartsev, A.; Sundström, V.; Scheblykin, I.G. Mechanistic Insights into Perovskite Photoluminescence Enhancement: Light Curing with Oxygen can Boost Yield Thousandfold. Phys. Chem. Chem. Phys. 2015, 17, 24978-24987.

${ }^{26}$ Tian, Y.; Merdasa, A.; Unger, E.; Abdellah, M.; Zheng, K.; McKibbin, S.; Mikkelsen, A.; Pullerits, T.; Yartsev, A.; Sundström, V.; Scheblykin, I. G. Enhanced OrganoMetal Halide Perovskite Photo-Luminescence from Nanosized Defect-Free Crystallites and Emitting Sites. J. Phys. Chem. Lett. 2015, 6, 4171-4177.

${ }^{27}$ deQuilettes, D. W.; Zhang, W.; Burlakov,V. M.; Graham, D. J.; Leitjens, T.; Osherov, A.; Bulovic, V.; Snaith, H. J.; Ginger, D. S.; Stranks, S. D. Photo-Induced Halide Redistribution in Organic-inorganic Perovskite Films. Nat. Commun. 2016, 7, 11683.

${ }^{28}$ Fang, H-H.; Adjokatse, S.; Wei, H.; Yang, J.; Blake, G.R.; Huang, J.; Even, J.; Loi, M.A. Ultrahigh Sensitivity of Methylammonium Lead Tribromide Perovskite Single Crystals to Environmental Gases. Sci. Adv. 2016, 2, e1600534.

${ }^{29}$ Handloser, K.; Giesbrecht, N.; Bein, T.; Docampo, P.; Handloser, M.; Hartschuh, A. Contactless Visualization of Fast Charge Carrier Diffusion in Hybrid Halide Perovskite Thin Films. ACS Photonics 2016, 3, 255-261.

${ }^{30} \mathrm{Fu}$, X.; Jacobs, D. A.; Beck, F. J.; Shen, H.; Catchpole, K. R.; White, T. P. Photoluminescence Study of Time-and Spatial-Dependent Light Induced Trap Deactivation in $\mathrm{CH}_{3} \mathrm{NH}_{3} \mathrm{PbI}_{3}$ Perovskite Films. Phys. Chem. Chem. Phys. 2016, 18, $22557-22564$. 
31 Fang, H-H.; Wang, F.; Adjokatse, S.; Zhao, N.; Loi, M.A. Photoluminescence Enhancement in Formamidinium Lead Iodide Thin Films. Adv. Funct. Mater. 2016, $26,4653-4659$.

${ }^{32}$ Mosconi, E.; Meggiolaro, D.; Snaith, H. J.; Stranks, S. D.; De Angelis, F. LightInduced Annihilation of Frenkel Defects in Organolead Halide Perovskites. Energy Environ. Sci. 2016, 9, 3180-3187.

${ }^{33}$ Brenes, R.; Guo, D.; Osherov, A.; Noel, N. K.; Eames, C.; Hutter, E. M.; Pathak, S. K.; Niroui, F.; Friend, R. H.; Islam, M. S.; et al. Metal Halide Perovskite Polycrystalline Films Exhibiting Properties of Single Crystals. Joule 2017, 1, $155-167$.

${ }^{34}$ Hoke, E.T.; Slotcavage, D.J.; Dohner, E.R.; Bowring, A.R.; Karunadasa, H.I.; McGehee, M.D. Reversible Photo-Induced Trap Formation in Mixed Halide Hybrid Perovskites for Photovoltaics. Chem. Sci. 2015, 6, 613-617.

${ }^{35}$ Saliba, M.; Matsui, T.; Seo, J-Y.; Domanski, K.; Correa-Baena, J-P.; Nazeeruddin, M. K.; Zakeeruddin, S. M.; Tress, W.; Abate, A.; Hagfeldt, A.; Grätzel, M. Incorporation of rubidium cations into perovskite solar cells improves photovoltaic performance. Science 2016, 354, 206-209.

${ }^{36}$ Saliba, M.; Matsui, T.; Seo, J-Y.; Domanski, K.; Seo J-Y.; Ummadisingu, A.; Zakeeruddin, S. M.; Correa-Baena, J-P.; Tress, W.; Abate, A.; Hagfeldt, A.; Grätzel, M. Cesium-containing triple cation perovskite solar cells: improved stability, reproducibility and high efficiency. Energy Environ. Sci. 2016, 9, 1989-1997.

${ }^{37}$ Saidaminov, M. I.; Kim, J.; Jain, A.; Quintero-Bermudez, R.; Tan, H.; Long, G.; Tan, F.; Johnston, A.; Zhao, Y.; Voznyy, O.; et al. Suppression of Atomic Vacancies via 
Incorporation of Isovalent Small Ions to Increase the Stability of Halide Perovskite Solar Cells in Ambient Air. Nat. Energy. 2018, 3, 648-654.

${ }^{38}$ Abdi-Jalebi, M.; Andaji-Garmaroudi, Z.; Cacovich, S.; Stavrakas, C.; Philippe, B.; Richter, J. M.; Alsari, M.; Booker, E. P.; Hutter, E. M.; Pearson, A. J.; et al. Maximizing and Stabilizing Luminescence from Halide Perovskites with Potassium Passivation. Nature 2018, 55, 497-501.

${ }^{39}$ Brenes, R.; Guo, D.; Osherov, A.; Noel, N. K.; Eames, C.; Hutter, E. M.; Pathak, S. K.; Niroui, F.; Friend, R. H.; Islam, M. S.; et al. Metal Halide Perovskite Polycrystalline Films Exhibiting Properties of Single Crystals. Joule 2017, 1, $155-167$.

${ }^{40}$ Meggiolaro, D.; Mosconi, E.; De Angelis, F. Mechanism of Reversible Trap Passivation by Molecular Oxygen in Lead-Halide Perovskites. ACS Energy Lett. 2017, 2, 2794-2798.

${ }^{41}$ Anaya, M.; Galisteo-López, J. F.; M. E. Calvo, J. P. Espinós, H. Míguez. Origin of Light Induced Ion Migration in Organic Metal Halide Perovskites in the Presence of Oxygen. J. Phys. Chem. Lett. 2018, DOI: 10.1021/acs.jpclett.8b01830

${ }^{42}$ Zhang, W.; Peng, L.; Liu, J.; Tang, A.; Hu, J-S.; Yao, J.; Zhao, Y.S. Controlling the Cavity Structures of Two-Photon-Pumped Perovskite Microlasers Adv. Mater. 2016, $28,4040-4046$.

${ }^{43}$ Tress, W.; Marinova, N.; Moehl, T.; Zakeerudin, S.M.; Nazeerudin, M.K.; Grätzel, M. Understanding the Rate-Dependent J-V Hysteresis, Slow Time Component, and Aging in $\mathrm{CH}_{3} \mathrm{NH}_{3} \mathrm{PbI}_{3}$ Perovskite Solar Cells: the Role of a Compensated Electric Field. Energy Environ. Sci. 2015, 8, 995-1004. 
${ }^{44}$ Deng, Y.; Xiao, Z.; Huang, J. Light-Induced Self-Poling Effect on Organometal Trihalide Perovskite Solar Cells for Increased Device Efficiency and Stability. $A d v$. Energy. Mater. 2015, 5, 1500721.

${ }^{45}$ Eames, C.; Frost, J. M.; Narnes, P. R. F.; O’Reagan, B. C.; Walsh, A.; Islam, S. Ionic Transport in Hybrid Lead Iodide Perovskite Solar Cells. Nat. Commun. 2015, 5, 7497.

${ }^{46}$ Deng, X.; Wen, X.; Lau, C. F. J.; Young, T.; Yun, J.; Green, M. A.; Huang, S.; HoBaillie, A. W. Y. Electric field induced reversible and irreversible photoluminescence responses in methylammonium lead iodide perovskite. J. Mater. Chem. C, 2016,

4, 9060-9068.

${ }^{47}$ Li, C.; Guerrero, A.; Zhong, Y.; Gräser, A.; Melo Luna,C.A.; Köhler, J.; Bisquert, J.; Hildner, R.; Huettner, S. Real-Time Observation of Iodide Ion Migration in Methylammonium Lead Halide Perovskite, Small 2017, 1701711.

${ }^{48}$ Li, C.; Guerrero, A.; Huettner, S.; Bisquert, J. Unravelling the role of vacancies in lead halide perovskite through electrical switching of photoluminescence. Nat. Commun. 2018, 9, 5113.

${ }^{49}$ Buin, A.; Comin, R.; Xu, J.; Ip, A. H.; Sargent, E. H. Halide-Dependent Electronic Structure of Organolead Perovskite Materials. Chem. Mater. 2015, 27, 4405-4412.

50 Tiede, D.O.; Calvo, M. E.; Galisteo-López, J. F.; Míguez, H. Improving the Bulk Emission Properties of $\mathrm{CH} 3 \mathrm{NH} 3 \mathrm{PbBr} 3$ by Modifying the Halide-Related Defect Structure. J. Phys. Chem. C, 2018, 122, 27250-27255.

${ }^{51}$ Kim, G. Y.; Senocrate, A.; Yang, T-Y.; Gregori, G.; Grätzel, m.; Maier, J. Large Tunable Photoeffect on Ion Conduction in Halide Perovskites and Implications for Photodecomposition. Nature Mater. 2018, 17, 445-449. 
52 Johnson, R. A. and Orlov, A. N. Physics of Radiation Effects in Crystals; Elsevier: Amsterdam, The Netherlands, 1986.

${ }^{53}$ Grancini, G.; D’Innocenzo, V.; Dohner, E. R.; Martino, N.; Srimath Kandada, A. R.; Mosconi, E.; De Angelis, F.; Karunadasa, H. I.; Hoke, E. T.; Petrozza, A. $\mathrm{CH}_{3} \mathrm{NH}_{3} \mathrm{PbI}_{3}$ Perovskite Single Crystals: Surface Photophysics and their Interaction with the Environment. Chem. Sci. 2015, 6, 7305.

${ }^{54}$ Yuan, Y.; Huang, J. Ion Migration in Organometal Trihalide Perovskite and its Impact on Photovoltaic Efficiency and Stability. Acc. Chem. Res. 2016, 49, 286-293.

${ }^{55}$ Gottesman, R.; Haltzi, E.; Gouda, L.; Tirosh, S.; Buhadana, Y.; Zaban, A. Extremely Slow Photoconductivity Response of $\mathrm{CH}_{3} \mathrm{NH}_{3} \mathrm{PbI}_{3}$ Perovskites Suggesting Structural Changes under Working Conditions. J. Phys. Chem. Lett. 2014, 5, 2662-2669.

${ }^{56}$ Zhou, Y.; You, L.; Wang, S.; Ku, Z.; Fan, H.; Schmidt, D.; Rusydi, A.; Chang, L.; Wang, L.; Ren, P.; Chen, L.; Yuan, G.; Chen, L.; Wang, J. Giant photostriction in organic-inorganic lead halide perovskites. Nat. Commun. 2016, 7, 11193.

${ }^{57}$ Tsai, H.; Asadpour, R.; Blancon, J.-C.; Stoumpos, C. C.; Durand, O.; Strzalka, J. W.; Chen, B.; Verduzco, R.; Ajayan, P. M.; Tretiak, S.; Even, J.; Alam, M. A.; Kanatzidis, M. G.; Nie, W.; Mohite, A. D. Light- Induced Lattice Expansion Leads to HighEfficiency Perovskite Solar Cells. Science 2018 , 360 ,67-70. 